\title{
Repercussões perinatais do Oligoidrâmnio na gestação de alto rissco
}

\author{
Perinatal repercussions of Oligohydraneum in high risk pregnancy \\ Repercusiones perinatales de Oligohidraneo en embarazo de alto riesgo
}

\begin{abstract}
RESUMO
Objetivo: Analisar os resultados perinatais da oligoidramnia em gestações de alto risco. Método: Estudo epidemiológico, observacional, retrospectivo, com abordagem quantitativa, realizado a partir de dados secundários de 3.448 gestantes de alto risco. Desse total, 44 gestantes tinham como intercorrência clínica a oligoidramnia. Os dados foram coletados no período de novembro de 2016 a outubro de 2017. A variável independente foi o oligoidrâmnio. As variáveis desfechos foram: prematuridade, baixo peso ao nascer, Apgar, óbitos fetais e neonatais e tipo de parto. Resultados: 0 Oligoidrâmnio apresentou associação estatística significativa com o parto pré-termo ( $p=0,05)$, o BPN ( $p<0,001)$, o Apgar reduzido no $1^{\circ}$ minuto $(p=0,03)$ e a morte infantil $(p=0,01)$. Conclusão: As oligoidramnia na gestação de alto risco associa-se a resultados perinatais desfavoráveis como prematuridade, BPN, Apgar reduzido no $1^{\circ} \mathrm{e} 5^{\circ}$ minuto e morte infantil, evidenciando a necessidade de cuidados especializados à gestante, por meio de pré-natal especializado e de qualidade. DESCRITORES: Saúde Materno-Infantil; Complicações na Gravidez; Oligoidrâmnio.
\end{abstract}

\section{ABSTRACT}

Objective: To analyze the perinatal results of oligohydramnias in high-risk pregnancies. Method: Epidemiological, observational, retrospective study, with a quantitative approach, carried out using secondary data from 3,448 high-risk pregnant women. Of this total, 44 pregnant women had oligohydramnias as a clinical complication. Data were collected from November 2016 to October 2017. The independent variable was oligohydramnios. The outcome variables were: prematurity, low birth weight, Apgar, fetal and neonatal deaths and type of delivery. Results: Oligohydramnios showed a statistically significant association with preterm birth ( $p=0.05)$, LBW (p $<0.001)$, reduced Apgar in the first minute $(p=0.03)$ and infant death $(p=0.01)$. Conclusion: Oligohydramnias in high-risk pregnancies are associated with unfavorable perinatal outcomes such as prematurity, LBW, reduced Apgar score in the 1st and 5th minutes and infant death, highlighting the need for specialized care for pregnant women, through specialized prenatal care and quality.

DESCRIPTORS: Maternal and Child Health; Pregnancy Complications; Oligohydramnios.

\section{RESUMEN}

Objetivo: analizar los resultados perinatales de oligohidramnias en embarazos de alto riesgo. Método: Estudio epidemiológico, observacional, retrospectivo con enfoque cuantitativo, realizado con datos secundarios de 3,448 embarazadas de alto riesgo. De este total, 44 mujeres embarazadas tenían oligohidramnias como complicación clínica. Los datos se recopilaron de noviembre de 2016 a octubre de 2017. La variable independiente fue oligohidramnios. Las variables de resultado fueron: prematuridad, bajo peso al nacer, Apgar, muertes fetales y neonatales y tipo de parto. Resultados: el oligohidramnios mostró una asociación estadísticamente significativa con el parto prematuro ( $p=0.05)$, LBW ( $<0.001$ ), Apgar reducido en el primer minuto ( $p=0.03$ ) y muerte infantil ( $p$ $=0.01$ ). Conclusión: Las oligohidramnias en embarazos de alto riesgo se asocian con resultados perinatales desfavorables como prematuridad, BPN, puntaje de Apgar reducido en el primer y quinto minutos y muerte infantil, lo que pone de relieve la necesidad de atención especializada para mujeres embarazadas, a través de atención prenatal especializada y calidad.

DESCRIPTORES: Salud Materno Infantil; Complicaciones del embarazo; Oligohidramnios.

RECEBIDO EM: 11/07/2020 APROVADO EM: 17/07/2020

\section{Renato Dias de Freitas}

Graduando em Medicina, Centro Universitário de Maringá (UNICESUMAR).

ORCID: 0000-0002-9124-2739 
Jefferson Dantas Lucena

Graduando em Medicina, Centro Universitário de Maringá (UNICESUMAR).

ORCID: 0000-0002-3378-8055

\section{Natália Lumi Ogassawara}

Graduanda em Medicina, Centro Universitário de Maringá (UNICESUMAR).

ORCID: 0000-0002-5539-0996

\section{Thaís Pordeus Leite Costa Mendes}

Médica. Especialista em Urgência e Emergência pela Faculdade Metropolitana do Vale do Aço (FAMEV).

ORCID: 0000-0003-3969-5877

\section{Pedro Bregola de Barros}

Médico. Especialista em Urgência e Emergência pela Faculdade Metropolitana do Vale do Aço (FAMEV).

ORCID: 0000-0001-6411-4902

\section{Sandra Marisa Pelloso}

Enfermeira. Doutora em Enfermagem pela Universidade de São Paulo (USP). Docente do Programa de Pós Graduação em Ciências da Saúde e do Programa de Pós Graduação em Enfermagem da Universidade Estadual de Maringá (UEM).

ORCID: 0000-0001-8455-6839

\section{Marcos Benatti Antunes}

Enfermeiro. Doutor em Enfermagem pela Universidade Estadual de Maringá (UEM). Docente do Curso de Medicina do Centro Universitário de Maringá (UNICESUMAR).

ORCID: 0000-0002-5139-8827

\section{INTRODUÇÃO}

A gestação é um processo fisiológico que culmina em muitas mudanças fisiológicas na mulher, contudo, muitas vezes podem agravar condições preexistentes ou desenvolver novas patologias e/ou situações que as tornam gestantes de alto risco, a exemplo, o oligoidrâmnio $^{(1)}$.

O oligoidrâmnio pode ser conceituado como a redução acentuada da quantidade do volume de líquido amniótico $(<500 \mathrm{ml})$, caracterizado como uma intercorrência clínica na gestação, tornando a mesma uma gestação de alto risco ${ }^{(2,3)}$.

Ao analisar na literatura os dados epidemiológicos de saúde relacionados ao oligoidrâmnio, a população mundial apresenta uma prevalência de cerca de 3,9 a 5,5 \% das gestações com essa comorbidade, e no Brasil, dos casos registrados no ano de 2012, observa-se uma incidência de 0,5 a 5,5 \% de gestações que apresentavam disfunção na quantidade de líquido amniótico ${ }^{(4,5)}$.

Essa patologia gera consequências significantes para a gestante e o neonatal, e quanto mais cedo o oligoidrâmnio se ins- tala, pior é o prognóstico da enfermidade. Um dos principais problemas para o feto é o crescimento intrauterino restrito (CIR) decorrente da baixa produção de líquido amniótico e mal funcionamento renal do feto. E é visto que os bebês que nascem decorrente dessa intercorrência clínica na gestação, apresentam-se pequenos para idade gestacional (PIG); com peso abaixo do $10^{\circ}$ percentil para a idade gestacional, além de estarem mais pré-dispostos a comorbidades vasculares (20 a 30\%), doenças metabólicas e do sistema nervoso ( 5 a $10 \%)$ e mortalidade perinatal, com cerca de dez vezes mais comparado com uma gestação de risco habitual ${ }^{(6)}$.

Outro estudo aponta repercussões da oligodramnia com resultados estatísticos significativos, destacando o tipo de parto $(\mathrm{p}<0,0002 ; \mathrm{RR}=0,32)$, sofrimento fetal agudo ( $\mathrm{p}<0,0004 ; \mathrm{RR}=2,2)$ e presença de malformações fetais $(p<0,01 ; R R=5,4)$. Além disso, os percentuais de malformações fetais foram de 17,6 e 3,3\% ${ }^{(7)}$.

Levando em consideração esses dados, o objetivo desse estudo foi analisar os resultados perinatais da oligoidramnia em gestações de alto risco.

\section{MÉTODO}

Trata-se de um estudo epidemiológico, observacional e retrospectivo, com abordagem quantitativa, realizado a partir de dados secundários de 3.448 prontuários de gestantes de alto risco. Desse total, 44 gestantes tinham como intercorrência clínica na atual gestação a oligoidramnia.

Os dados foram coletados no período de novembro de 2016 e outubro de 2017 , por meio das informações contidas nos prontuários das gestantes, formulário de classificação de risco e livro de registro de recém-nascidos do hospital de referência ao parto.

A variável independente foi a intercorrência clínica de oligoidrâmnio. As variáveis desfechos foram: prematuridade, baixo peso ao nascer (BPN), Apgar $<7$ no $1^{\circ}$ e $5^{\circ}$ minutos, óbito fetal, óbito neonatal e tipo de parto (Normal e Cesária).

Os dados foram digitados e organizados em uma planilha eletrônica da Microsoft Office Excel $2017^{\circ}$, posteriormente processamento e analisados com o software Epi Info 7.0, programa de domínio público. Os dados foram submetidos aos testes 
Qui-quadrado (x2) e Teste Exato de Fisher em nível de 5\% de significância $(\mathrm{p}<0,05)$ e intervalo de confiança (IC) de $95 \%$.

Foram atendidas todas as normas de Pesquisas Envolvendo Seres Humanos do
Conselho Nacional de Saúde (resolução CNS 466/2012) e foi aprovada conforme parecer $n^{\circ}$ 2.287.476 do Comitê Permanente de Ética em Pesquisa com Seres Humanos (COPEP).

Tabela 1 - Perfil sociodemográfico das gestantes estratificadas como alto risco com e sem oligoidrâmnio como Intercorrência Clínica na Atual Gestação. Maringá, PR - Brasil, 2019.

\begin{tabular}{|c|c|c|c|c|c|}
\hline \multirow{2}{*}{ Variáveis } & \multicolumn{2}{|c|}{ Sem Oligoidrâminio } & \multicolumn{2}{|c|}{ Com Oligoidrâmnio } & \multirow{2}{*}{ p } \\
\hline & $\mathbf{N}$ & $\%$ & $\mathbf{N}$ & $\%$ & \\
\hline Idade & & & & & 0,19 \\
\hline 10 a 19 & 271 & 97,8 & 6 & 2,2 & \\
\hline 20 a 34 & 2376 & 98,7 & 32 & 1,3 & \\
\hline 35 ou mais & 757 & 99,2 & 6 & 0,8 & \\
\hline Cor & & & & & 0,71 \\
\hline Branca & 1959 & 98,8 & 23 & 1,2 & \\
\hline Preta & 147 & 99,3 & 01 & 0,7 & \\
\hline Parda & 1292 & 98,5 & 20 & 1,5 & \\
\hline Amarela & 6 & 100,0 & - & - & \\
\hline Situação conjugal & & & & & 0,69 \\
\hline Vive com o companheiro & 1957 & 98,8 & 24 & 1,2 & \\
\hline Não vive com o companheiro & 1447 & 98,6 & 20 & 1,4 & \\
\hline Escolaridade & & & & & 0,91 \\
\hline$<8$ anos & 1653 & 98,8 & 21 & 1,2 & \\
\hline$\geq 8$ anos & 1751 & 98,7 & 23 & 1,3 & \\
\hline Trabalho remunerado & & & & & 0,35 \\
\hline Sim & 1932 & 98,6 & 28 & 1,4 & \\
\hline Não & 1472 & 98,9 & 16 & 1,1 & \\
\hline Fonte: Dados da pesquisa, 2020. & & & & & \\
\hline
\end{tabular}

Tabela 2 - Associação entreOligoidrâmnio e resultados perinatais de gestação de alto risco, Maringá, PR - Brasil, 2020.

\section{Resultados Perinatais}

Oligoidrâmnio ( $n=44)$

\begin{tabular}{lccc}
\multicolumn{1}{c}{ Resultados Perinatais } & N & $\%$ & P \\
Prematuridade (<37 semanas) & 20 & 45,5 & 0,05 \\
Baixo peso ao nascer (<2.500 g) & 18 & 40,9 & $<0,001$ \\
Apgar $1^{\circ}$ min. (<7) & 13 & 29,6 & 0,03 \\
Apgar 5 ${ }^{\circ}$ min. (<7) & 06 & 13,6 & 0,02 \\
Morte fetal & 01 & 2,3 & $0,30^{*}$ \\
Morte infantil & 04 & 9,1 & $0,01^{*}$ \\
Cesárea & 35 & 79,6 & 0,31 \\
Fonte:Dados da pesquisa, 2020. & & &
\end{tabular}

\section{RESULTADOS}

Das 3.448 gestantes atendidas no ambulatório de alto risco, 44 (1,28\%) tiveram como risco o oligoidrâmnio, dessas as características predominantes foram: idade entre 20 e 34 anos (72,7\%), pele branca $(52,3 \%)$; vivem com o companheiro $(54,5 \%)$; escolaridade igual ou superior a 8 anos (52,3\%); e trabalho remunerado (63,6\%), conforme apresenta a tabela 1 .

A tabela 2 apresenta o Oligoidrâmnio como Intercorrência Clínica na Atual Gestação com associação estatística significativa com o parto pré-termo $(\mathrm{p}=0,05)$, o $\mathrm{BPN}$ $(\mathrm{p}<0,001)$, o Apgar reduzido no $1^{\circ}$ minuto $(\mathrm{p}=0,03)$ e a morte infantil $(\mathrm{p}=0,01)$.

\section{DISCUSSÃO}

Observando as literaturas mais atuais, nota-se que cerca de $4 \%$ das complicações perinatais são geradas por oligoidramnia. $\mathrm{E}$ as principais consequências vistas são: parto prematuro, apgar baixo, no primeiro e quinto minuto, além de acentuados problemas respiratórios no momento do nascimento, como síndrome da angústia respiratória (SARA), pois geralmente o pulmão desse bebê não se encontra maturado adequadamente. Acredita-se que os pneumócitos do tipo II não produzem líquido surfactante de forma adequada, devido à desnutrição causada pela patologia, ou mesmo não diagnóstico da enfermidade em alguns dos trimestres, ficando limitado o uso de métodos preventivos, como os corticoides utilizados para auxiliar na maturação pulmonar até $34^{\circ}$ semana ${ }^{(4,6)}$.

Ao analisar os dados epidemiológicos deste estudo realizado com gestações de alto risco por intercorrências clínicas na atual gestação, especificamente por oligoidrâmnio, nota-se a permanência de problemas para o feto e a gestante, como parto prematuro como principal complicação, seguido de baixo peso para a idade gestacional, Apgar baixo, e mais raramente morte materna e neonatal. Este último relacionado a qualidade de saúde regional, visto que os municípios analisados apresentam padrão acima da média com rela- 
ção a atenção a saúde materno-infantil.

Quando se observa os resultados do município de Maringá/PR, no geral, percebe-se que o padrão de apresentação da doença é cerca de 3\% menor, quando comparado a outros locais da América. E no Brasil, em avaliação feita em Pernambuco com gestações de alto risco, limiar de idade e quantidade de anos de estudo semelhantes, no período de 2007 e 2008, também obteve dados de prevalência parecidos com as referências bibliográficas, e com o Equador por exemplo. Deste modo, observando o perfil epidemiológico das gestantes do noroeste do Paraná, nota-se um maior número de anos de estudo, e mais gestantes com mais de 20 anos, e isso aparentemente, contribui para uma menor permanência da instalação da doença, e melhores prognósticos perinatais ${ }^{(4)}$.

Ainda, com relação a idade materna, ocorre íntima associação do oligoidrâmnio com a idade materna, nota-se que nos países desenvolvidos ocorre um maior acometimento do público adulto jovem. Algumas literaturas fazem uma relação com o estresse vivido por essas mulheres, em que a maioria já está inserida no mercado de trabalho, e tem metas para cumprir, o que geraria um possível desequilíbrio hormonal e hidroeletrolítico na gestante $^{(8)}$. Na presente pesquisa, o padrão de manifestação tem se mostrado contrário, se observa uma maior prevalência nas gestantes de 10 a 19 anos, público adolescente predominantemente. $\mathrm{O}$ que sugere uma relação maior com menos anos de estudo, e baixa quantidade de mulheres inseridas no mercado de trabalho. Além disso, o que se espera é que esses tipos de gestações não sejam planejadas, e pela própria idade se deduz que essas mulheres não possuam companheiro fixo. Esses fatos seriam fatores de risco importantes para o desencadeamento de outras patologia ${ }^{(8)}$.

Ao analisar as etiologias do quadro clínico de oligohidrâmnios, é possível observar que doenças clínicas e hipertensivas são responsáveis por mais da metade dos casos de oligoidramnia, cerca de $54 \%$, e outros $41,5 \%$ causados por rotura prematura de membranas. Uma das expli-
Ainda, com relação a

idade materna, ocorre

íntima associação do

oligoidrâmnio com

a idade materna,

nota-se que nos

países desenvolvidos

ocorre um maior

acometimento do

público adulto jovem.

Algumas literaturas

fazem uma relação

com o estresse vivido

por essas mulheres, em

que a maioria já está

inserida no mercado

de trabalho, e tem

metas para cumprir,

o que geraria um

possível desequilíbrio

hormonal e

hidroeletrolítico

na gestante. cações para isso é que decorrente dessas anormalidades, pode-se desencadear uma insuficiência uteroplacentária, a qual pode restringir o crescimento fetal e diminui o débito urinário. Quando a etiologia é por anormalidades fetais, a principal causa é anomalia geniturinária. Dentre essas anomalias, pode se observar agenesia renal bilateral, rins displásicosmulticísticos e estenose uretral ${ }^{(4,8)}$.

$\mathrm{Na}$ presente análise, ocorre uma predominância de casos de oligoidramnia em mulheres declaradamente brancas e pardas. Isso é comprovado pelo fato de apenas $0,6 \%$ de mulheres negras apresentarem oligoidramnia contra $1,2 \%$ e $1,5 \%$ do público referido a priori. Quando se observa um município como Maringá/PR é esperado esse padrão, já uma minoria de indivíduos se considera negra nos censos do IBGE, menos de $10 \%$, e também pelo espectro de colonização regional que é europeu caucasiano, como visto nos dados epidemiológicos ainda é mais predispostos a redução de líquido amniótico ${ }^{(9)}$.

Comparando os índices de parto prematuro ( $<37$ semanas), observa-se uma significativa ocorrência a nível mundial. Como no levantamento realizado, foi constatado que $45,5 \%$ dos casos de oligoidrâmnio tiveram parto prematuro, seguindo próximo a um padrão de ocorrência de $42 \%$ de parto prematuro espontâneo e $20 \%$ de parto prematuro indicado, em outros levantamentos. Isso associada a maioria das vezes ao baixo peso para idade gestacional e apgar baixo, esses eventos clínicos trazem consigo mais problemas como síndrome da angústia respiratória (SARA) e dificuldades de aleitamento. Além disso, se observa que bebês decorrentes de partos complicados e apgar baixo são mais predispostos aos atrasos do desenvolvimento neuro cognitivo, e doenças psiquiátricas como transtorno de déficit de atenção (TDAH) e transtorno do espectro autista ${ }^{(3,10,11)}$.

A taxa de mortalidade infantil decorrente de consequências do oligoidrâmnio se mostrou-se relativamente baixa no estudo, cerca de 9,1\%. Isso em decorrência do elevado números de partos cesarianos, 
visto também no estudo, cerca de $79 \%$ dos casos. Esse tipo de parto é indicado em gravidez de risco, como as que evoluem com oligoidrâmnio. No entanto, um dos principais problemas é com relação ao apgar baixo, que vai ocorrer tanto no primeiro como no quinto minuto, geralmente os neonatos apresentam uma taxa de recuperação mais lenta e requerem muitas vezes um leito que contenha UTI para conter as complicações da baixa adaptação ao ambiente. E isso é um grande problema, porque atualmente a região conta com baixíssimos locais que contém esse tipo de quarto, e muitas vezes a mortalidade se relaciona com a falta desse suporte ${ }^{(10)}$.

O acompanhamento pré-natal especializado em gestações de alto risco, como as provocadas pelo oligoidrâmnio é muito importante, e é preconizado monitoramento diferenciado pelo Ministério da Saúde, no que tange a pelo menos duas consultas ao mês, prescrição de dieta específica e corticoides. Além disso, a atenção primária e secundária devem compartilhar informações sobre a assistência prestada pela equipe multidisciplinar, a fim de garantir melhor qualidade do pré-
As oligoidramnia

na gestação de alto

risco associa-se a

resultados perinatais

desfavoráveis como

prematuridade,

BPN, Apgar

reduzido no $1^{\circ} \mathrm{e}$

$5^{\circ}$ minuto e morte

infantil... -natal habitual e especializado.Sugere-se visitas domiciliares em maior número, uma vez que contribuem positivamente para o psicológico da gestante, pois ela se sente mais acolhida e apoiada, consequentemente acaba cuidando melhor de sua gestação e evita desfechos como o aborto espontâneo ${ }^{(11,12,13)}$.

\section{CONCLUSÃO}

As oligoidramnia na gestação de alto risco associa-se a resultados perinatais desfavoráveis como prematuridade, BPN, Apgar reduzido no $1^{\circ}$ e $5^{\circ}$ minuto e morte infantil, evidenciando a necessidade de cuidados especializados à gestante, por meio de pré-natal especializado e de qualidade.

Diante a esses resultados é necessário reforçar a atenção da equipe multidisciplinar nesse tipo de gestação, uma vez que os desfechos de gestante de alto risco acompanhadas e monitoradas frequentemente, como recomendado pelo Ministério da Saúde, evoluem positivamente e evitam consequências como os desafetos psicológicos, síndromes hipertensivas e desequilíbrios fisiológicos propriamente ditos, evitando agravos no pré-parto e pós parto. -

\section{REFERÊNCIAS}

1. Melo WA, Alves Jl, Ferreira AAS, Maran E. Gestação de alto risco: fatores associados em município do noroeste paranaense. Espaço para a saúde - Revista de saúde pública do Paraná. 2016; 17:8291.

2. Freire DM, Cecatti JG, Paiva CS. Is uterine height able to diagnose amniotic fluid volume deviations. Rev Bras Ginecol Obstet. 2013;35(2):49-54.

3. Paraná. Secretaria Estadual de Saúde. Linha guia: mãe paranaense. Curitiba: SESA, 2018. 63 p.

4. Souza ASR, et al. Desfechos maternos e perinatais em gestantes com líquido amniótico diminuído. RevBrasGinecol Obstet. 2013; 35(8):342-8.

5. Rabie N, et al. Oligohydramnios in complicated and uncomplicated pregnancy: a systematic review and meta analysis. Ultrasound Obstet Gynecol. 2017; 49(4):442-449.

6. Rezende J, Montenegro CAB. Obstetrícia. $12^{\text {a }}$ edição. Rio de Janeiro: Guanabara Koogan, 2013.

7. Madi JM, et al. Oligodramnia sem rotura das membranas am- nióticas: resultados perinatais. Rev. Bras. Ginecol. Obstet. 2005; 27( 2 ): 75-79.

8. Gary F, Leveno KJ. Manual de Obstetrícia de Williams: complicações na gestação, 23ª edição. São Paulo: McGraw-Hill, 2014.

8. IBGE. Autodeclaração de cor e raça. Maringá, Paraná: 2010. Disponível em: https://cidades.ibge.gov.br/brasil/pr/maringa/pesquisa/23/25888?detalhes=true. Acesso em: 01/07/2020.

9. Zugaib M. Obstetrícia. $3^{\text {a }}$ ed. Barueri, São Paulo: Manole, 2016.

10. Kaplan HI, Sadock B. Compêndio de Psiquiatria. $11^{\text {a }}$ edição. Porto Alegre: Artmed, 2017.

11. Brasil.Manual Técnico: Gestação de Alto Risco. Ministério da Saúde. Secretaria de Atenção à Saúde.Departamento de Ações Programáticas Estratégicas. - Brasília: Ministério da Saúde, 2012. Disponível em: https://bvsms.saude.gov.br/bvs/publicacoes/manual_tecnico_gestacao_alto_risco.pdf. Acesso em: 01/07/2020.

12. Fradin AB.Gestação de Risco: proposta de intervenção para um planejamento no Programa Saúde da Família. Canaã. Minas Gerais. 2017. 